\title{
Efficacy of Symbiotics in an Experimental Model of Endotoxic Shock Induced by Lipopolysaccharide from Escherichia Coli
}

Erika D. Gonzalez-Renovato ${ }^{1}$, Genaro Gabriel Ortiz ${ }^{*}$, Fermin P. Pacheco-Moises ${ }^{2}$, Jose A. Cruz-Serra$n^{3}$, Alfredo de J. Celis de la Rosa ${ }^{4}$, Oscar K. Bitzer-Quintero ${ }^{5}$, Angelica L. Sanchez Lopez ${ }^{1}$ and Moises A. Alatorre-Jimenez ${ }^{1}$

${ }^{1}$ Laboratory of Development, Aging and Neurodegenerative Diseases, Neurosciences Division, Biomedical Research Centre West (CIBO), Mexican Social Security Institute, Sierra Mojada 800, Col. Independence, 44340 Guadalajara, Jalisco, Mexico

${ }^{2}$ Department of Chemistry, University Center of Exact Sciences and Engineering, University of Guadalajara, Boulevard Gral, Marcelino Garcia Barragan 1421, Olympic, 44430 Guadalajara, Jalisco, Mexico

${ }^{3}$ Research Coordinator and Director General Company Kurago Biotech SA de CV, Moctezuma \# 144 Zapopan, Jalisco, Mexico ${ }^{4}$ Department of Public Health. University Center for Health Sciences, East Independence, 44340 Guadalajara, Jalisco, Mexico ${ }^{5}$ Immunomodulation Laboratory Division of Neuroscience, Biomedical Research Centre West (CIBO), Mexican Social Security Institute, Sierra Mojada 800, Col. Independence; 44340 Guadalajara, Jalisco, Mexico

*Corresponding author: Dr. Genaro Gabriel Ortíz, Development- Aging Laboratory, Neurodegenerative diseases, Division of Neurosciences, CIBO-IMSS, Sierra Mojada 800 CP 44340. Guadalajara, Jalisco, México, Tel: (0133) 2015 3651;

E-mail: genarogabriel@yahoo.com

\begin{abstract}
Obsevation: Endotoxic shock is a life-threatening condition and its sequelae represent an array of clinical symptoms that encompass systemic inflammation, coagulopathy, and abnormalities of the renal, hepatic, pulmonary and hematologic systems, followed by multi-organ failure. Furthermore, systemic failure leads to an unfavorable environment in the gut that causes an imbalance in the homeostasis of intestinal microbiota. In the present study, the effect of symbiotics as adjuvant therapy in endotoxic shock was investigated. Adult male Wistar rats were randomized into three groups: Control group received a single intraperitoneal injection of physiological saline solution, and the other two groups received either a symbiotic formulation or a placebo daily, for one week, followed by the administration of a single lethal dose of lipopolysaccharide (LPS) from E. coli $(20 \mathrm{mg} / \mathrm{Kg}$, IP).

Results: The symbiotic formulation used improved the survival rate of LPS-treated rats, ameliorated the clinical symptomatology, reduced the production of serum proinflammatory cytokines (TNF- $\alpha$, IL-6, IL-1 $\beta$ ), and preserved the mitochondrial membrane fluidity and ATPase activity.

Conclusion: The symbiotic formulation used as a pre-treatment in this experimental model, reduced mortality and showed beneficial effects at the systemic and sub-cellular levels.
\end{abstract}

Received Date: April 13, 2016

Accepted Date: June 06, 2016

Published Date: June 09, 2016

Citation: Gonzalez-Renovato, E.D., et al. Efficacy of Symbiotics in an Experimental Model of Endotoxic Shock Induced by Lipopolysaccharide from Escherichia Coli. (2016) Int J Food Nutr Sci 3(1): 252-258.

DOI: $10.15436 / 2377-0619.16 .865$

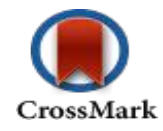

Keywords: Symbiotic; Proinflammatory cytokines; Endotoxic shock; Mitochondria; Membrane fluidity; Experimental study

\section{Introduction}

Endotoxic shock is the result of severe infection caused by gram-negative bacteria (GNB), is consider a common cause of death in intensive care units (ICU) ${ }^{[1]}$, and arises as a host's response to bacterial infection (Endotoxins such as lipopolysaccharide $($ LPS $))^{[2]}$. Sepsis and endotoxic shock affects about 700,000 people per year in the US alone, of which about 200,000 die $^{[3]}$. Therefore, the use of animal models is relevant in testing new drugs (Or dietary supplements) and techniques that cannot be tested in humans, for future development of novel therapies and/or treatments for endotoxic shock. 
When it is released in the body, LPS triggers a systemic infection that induces an acute state of metabolic-circulatory dysfunction leading to serious complications. Lipopolysaccharide is a complex amphipathic macromolecule found in the outer membrane of GNB, like Escherichia coli ${ }^{[4]}$, and it triggers the first line of defense in the organism. Liver Kupffer cells are major sources of production of tumor necrosis factor alpha (TNF- $\alpha$ ) and other pro-inflammatory mediators (Nitric oxide, superoxide, eicosanoids, interleukin-1 (IL-1), and interleukin-6 (IL-6)) that are excessively released into circulation; with possible alterations to the liver functions resulting in changes to the integrity of liver vasculature ${ }^{[5]}$ or on the regulation of liver cytochrome P450 contents and metabolic capacity ${ }^{[6]}$. Oxidative stress is a response to a microbial invasion. Molecules released during this process, such as reactive oxygen species (ROS) and nitric oxide (NO), play an important role in septic shock injury, mediating the inflammatory reaction. Although the link between oxidative stress and the inflammatory pathway has not been fully described, the release of cytokines and ROS constitute the markers of inflammation in septic shock ${ }^{[7]}$. Furthermore, this host-bacteria interaction may lead to an intestinal gut imbalance of the microbiota in favor of pathogens that results in a deregulated mucosal immune response and an inflammatory state in the host; as well as neutrophil recruitment and barrier dysfunction, inducing multiple organ dysfunction that underlies the pathogenesis of endotoxic shock ${ }^{[8-11]}$. It is well known that intestinal microbiota exert a positive influence on systemic homeostasis in the host, so a hostile environment in the gut can lead to further complications like an increase in the proinflammatory response and barrier dysfunction, leading to multiple organ dysfunction ${ }^{[5,10]}$.

The classical concept of nutrition, understood as the administration of calories, proteins and trace elements to maintain the function of the body, has become history ${ }^{[12]}$. Food is made up of nutrients not only understood as assailable substances that allow the body to get energy, build and repair tissues and regulate metabolic processes, but also understood as those substances that are able to affect physiological functions of the individual like the immune system, and play a pivotal role in the field of disease prevention ${ }^{[13,14]}$. Because of the advancement of science and the development of knowledge on the biochemistry found in foods, other ingredients can be associated to bring about proper functioning of the body. Research and the discovery of the different components of food and its specific function have openedup the possibility of a new concept of nutrition.

The use of probiotics and prebiotics in the treatment of bacterial infection has been called a promising intervention ${ }^{[9,11]}$ with favorable impact on mucosal immunity; so, nutritional supplementation with symbiotics may prevent damage and confer beneficial effects in the host through maintaining barrier function and immune defense by preventing the colonization of pathogens. The term symbiotic refers to the mixture that combines live beneficial microorganisms (probiotics) that restore the intestinal microbiota, and selective components that stimulate bacteria growth like oligosaccharide (prebiotics), modulating the immune response against infectious agents ${ }^{[10,15-17]}$. Moreover, symbiotics are capable of altering the composition of the colonic microbiota, reducing inflammatory processes in the gut mucosa. It has also been demonstrated that in patients undergoing surgery symbiotics are capable of prevent bacterial infections ${ }^{[18,19]}$.

Although the use of symbiotics exerts beneficial prop- erties and they are used in various clinical conditions, the mechanisms by which they act are still not entirely understood. And while the results are promising we must take into account that not all strains are equally beneficial, and mechanisms of action may differ in probiotics species. For this reason, the use and testing of different strains as potential for the treatment of different conditions is necessary.

Multi-organ failure/dysfunction syndrome correlates with poor outcome in endotoxic shock. Hence, in the present study we examined the protective effect of the administration of symbiotics on survival, serum pro-inflammatory cytokine levels, and biochemical parameters (Mitochondrial membrane fluidity and ATPase activity of liver, kidney and lung) in an experimental model of endotoxic shock induced by lipopolysaccharide from E. coli.

\section{Materials and Methods}

The symbiotic used was a commercial formulation containing: $1 \times 10^{11}$ colony-forming unit (CFU) of Lactobacillus rhamnosus (NH001) strain, 1x10 11 CFU of Bifidus bacterium (Bi-07) strain (As a probiotic), and $3 \mathrm{~g}$ of omega-3 fatty acids, fructooligosaccharides, and vitamin supplements (Vitamin E, folic acid, vitamin B12, ascorbic acid and pyridoxine hydrochloride) (As a prebiotic) (Table 1). The symbiotics and placebo were donated by KURAGO-BIOTEK ${ }^{\circledR}$ Corporation (Zapopan, Jalisco. Mexico). The LPS from E. coli, serotype 0111:B4 was purchased from Sigma Chemical Co. (St. Louis, MO. USA).

\section{Animals}

Adult male Wistar rats $(\mathrm{n}=50)$ with a weight range of $200-250 \mathrm{~g}$, were kept in standard laboratory conditions, in a $12 \mathrm{~h} / 12 \mathrm{~h}$ light-dark cycle, at $22 \pm 2^{\circ} \mathrm{C}$; fed a standard diet (Chow Purina), and water ad libitum. They were obtained from the Occidental Biomedical Centre- Mexican Social Security Institute (Centro de Investigación Biomédica de Occidente - Intituto Mexicano del Seguro Social (CIBO-IMSS)) in Guadalajara, Jalisco, Mexico. All animals received humane care in accordance with international guidelines on the ethical use of animals and the Official Mexican Guidelines known as the Norma Oficial Mexicana NOM-062-ZOO-1999. Animals were randomly assigned to three experimental groups as follows:

Group $1($ Control, $\mathbf{n}=\mathbf{1 0})$ : treated with a single dose $(10 \mu \mathrm{L})$ of physiologic isotonic saline solution administered by intraperitoneal (IP) injection.

Group 2 (Symbiotics plus LPS, $\mathbf{n}=\mathbf{2 0}$ ): were administered a symbiotic formulation daily for one week (Volume administered: $1 \mathrm{~mL}$ ) and subsequently received a single LD100 dose $(20 \mathrm{mg} / \mathrm{Kg}$ of body weight, IP)[20] of LPS from E. coli, strain 0111: B4. The symbiotic suspension was administered through an intragastric cannula.

Group 3 (Placebo plus LPS, $\mathbf{n}=\mathbf{2 0}$ ): were administered a placebo ( $1 \mathrm{~mL}$ of $10 \%$ sucrose) daily for one week, and subsequently received a single LD100 dose of $(20 \mathrm{mg} / \mathrm{Kg}$ of body weight, IP) of LPS. The placebo solution was administered through an intragastric cannula.

Experimental groups 2 and 3 were divided into experimental sub-groups for:

1) The determination of clinical symptomatology and survival rate, $(n=10)$. 
2) The determination of biochemical parameters (Quantification of pro-inflammatory cytokines, mitochondrial membrane fluidity, and ATPase activity), $(\mathrm{n}=10)$.

\section{Determination of survival}

Animals were kept under observation to assess for clinical symptomatology ( $\mathrm{n}=10$ per group). Clinical symptomatology (Hirsutism, adynamia, palpebral edema, and acute diarrhea) was evaluated every 15 minutes for 75 minutes. Then a Kaplan-Meier cumulative survival plot was constructed (Using SPSS version 19 for Windows).

\section{Biochemical assays}

After treatment rats were sacrificed by cervical dislocation. Blood was collected immediately and was centrifuged for 10 minutes at $3000 \mathrm{rpm}$ to collect the serum. Dissection of the tissues was performed immediately (Liver, kidney and lung) to isolate the mitochondria.

\section{Quantification of cytokines}

The TNF- $\alpha$, IL- $1 \beta$ and IL- 6 levels in serum were quantified using commercially available ELISA kits specific for rat TNF- $\alpha$ (Genzyme, Cambridge, MA, USA), IL-1 $\beta$ and IL-6 (Both from Endogen, Woburn, MA, USA). The sensitivity of the assays for TNF- $\alpha$, IL- $1 \beta$ and IL- 6 was $5 \mathrm{pg} / \mathrm{mL}, 12 \mathrm{pg} / \mathrm{mL}$ and $15 \mathrm{pg} / \mathrm{mL}$, respectively. Absorbance was measured at $405-414$ $\mathrm{nm}$ a maximum of $15 \mathrm{~min}$ after the addition of the substrate, using a UV-Vis spectrophotometer (BIO-RAD 550).

\section{Isolation of the mitochondria}

Tissues were washed in saline solution $(0.9 \% \mathrm{NaCl})$ to remove excess blood, and the mitochondria were isolated by differential gradient centrifugation. Briefly, the tissue was homogenized with a Teflon ${ }^{\circledR}$ pestle PYREX ${ }^{\circledR}$ Potter-Elvehjem tissue grinder in $20 \mathrm{~mL}$ of cold SHE buffer (250 mM sucrose, 25 $\mathrm{mM}$ Hepes ( $\mathrm{pH} 7.5)$ and $1 \mathrm{mM}$ EGTA) maintained in ice. Once obtained, the homogenate was centrifuged at $600 \mathrm{xg}$ for $10 \mathrm{~min}$ at $4^{\circ} \mathrm{C}$. The pellet was discarded, and the supernatant was centrifuged at $10000 \mathrm{xg}$ for $10 \mathrm{~min}$ at $4^{\circ} \mathrm{C}$. The foamy layer at the top of the supernatant was removed and the mitochondrial pellet was washed with SHE buffer (Containing $0.1 \%$ fatty acid free serum albumin). Then, the pellet was re-suspended in $2 \mathrm{~mL}$ of SHE buffer ${ }^{[21]}$.

Quantification of the mitochondrial protein was determined by a quantitative colorimetric process for proteins through the Folin reaction based on the Lowry method, in the presence of $0.066 \%$ sodium deoxycholate using bovine serum albumin as a standard: first the proteins are pre-treated with copper ion in alkali solution, and then, in the presence of the Folin-phenol reagent, a reduction reaction occurs turning the end-product of this reaction into a blue color. The amount of proteins in the sample can be read at an absorbance of $750 \mathrm{~nm}^{[22]}$.

\section{Membrane fluidity}

Mitochondrial membrane fluidity was estimated from the excimer to monomer fluorescence intensity ratio (Ie/Im) of the fluorescent probe 1,3 dipyrenylpropane (DPyP) incorporated in submitochondrial particles. Briefly, $0.25 \mathrm{mg}$ of mitochondrial protein and $0.1 \mathrm{nmol}$ DPyP were mixed with $10 \mathrm{mM}$ Tris- $\mathrm{HCl}$ buffer ( $\mathrm{pH}$ 7.8). The mixtures were incubated in darkness at $4^{\circ} \mathrm{C}$ for $5 \mathrm{~h}$, in order to achieve maximum incorporation of the DPyP to the membranes. The fluorophore was excited at $329 \mathrm{~nm}$ and the monomer and excimer fluorescence intensities were read at 379 and $480 \mathrm{~nm}$, respectively. From these readings the excimer to monomer fluorescence intensity ratio (Ie/Im) was calculated. Membrane fluidity was expressed as a fluorescence intensity ratio of excimer and monomer DPyP (Ie/Im ratio), and a high Ie/ Im ratio indicates higher membrane fluidity. Fluorescence was measured at $24^{\circ} \mathrm{C}$ on a Perkin Elmer fluorescence spectrometer LS50B, and for sample analysis the FL WinLab 3.0 software was used, as recommended by the manufacturer. Fluorescence corrections obtained from readings of membranes without DPyP were applied to all fluorescence values ${ }^{[23]}$.

\section{Mitochondrial ATPase activity}

ATPase activity was determined by quantification of the inorganic phosphate (PO4) released, using the colorimetric method described by Sumner ${ }^{[24]}$. The standard reaction medium (1 mL) contained: $125 \mathrm{mM} \mathrm{KCl}, 40 \mathrm{mM}$ Hepes/KOH (pH 8.0), $0.1 \mathrm{mM}$ EGTA, $3 \mathrm{mM}$ ATP, and $5 \mathrm{mM} \mathrm{MgCl}_{2}$. The reaction was initiated by the addition of a mitochondrial fraction $(1 \mathrm{mg}$ of protein) and quenched by the addition of $100 \mu \mathrm{L}$ of cold trichloroacetic acid $30 \%(\mathrm{w} / \mathrm{v})$. Afterwards, the sample was centrifuged for $10 \mathrm{~min}$ at $3,500 \mathrm{rpm}$; and $800 \mu \mathrm{L}$ of the supernatant was separated, and $1 \mathrm{~mL}$ of $3.3 \%$ ammonium molybdate was added, followed by $100 \mu \mathrm{L}$ of $10 \%$ ferrous sulphate. The reading of the absorbance of the samples was recorded at $660 \mathrm{~nm}$ using a Benchmark Plus Microplate Spectrophotometer System, 110/230 V by Bio-Rad.

\section{Statistical analysis}

The assessments of clinical symptomatology and differences in survival rate between groups were evaluated using an individualized tracking program in response to endotoxic shock, and somatic parameters and behavioral responses were analyzed. Survival curves (Kaplan-Meier) were made and evaluated in accordance with the software SPSS ver. 19 for Windows. Proinflammatory cytokine levels in serum were analyzed using a non-parametric test (Kruskal-Wallis), followed by the Mann-Whitney U test. Mitochondrial membrane fluidity and ATPase activity were evaluated using parametric tests: the oneway analysis of variance (ANOVA), followed by multiple comparison between groups (Newman-Keul's student T). The level of significance was $p<0.05$. Values are expressed as means \pm SEM.

\section{Results}

\section{Effects of symbiotic supplementation on pathophysiological responses}

In the first part of the experimental study we assessed the animals with an individualized tracking program in response to endotoxic shock. Figure 1 shows pathophysiological response in the rats under the treatment of Placebo plus LPS (P + LPS). Hirsutism was evident in this group of rats at shorter times (45 minutes) after the induction of shock. Acute diarrhea and adynamia were presented in $100 \%$ of rats at minute 75 . Furthermore, palpebral edema was registered at 45 minutes and at 75 minutes after the induction of shock in $90 \%$ of the animals. In contrast, rats under the treatment of Symbiotic plus LPS (S + 
LPS) showed a significant decrease in symptoms compared to placebo treated rats, and the clinical findings were seen at later times (Figure 1). Namely, hirsutism was registered in four rats at 60 minutes, and in eight rats at 75 minutes; while, palpebral edema and acute diarrhea presented at 45 minutes $(n=1)$ and 60 minutes $(n=2)$, respectively, and $60 \%$ of the animals presented with these conditions at 75 minutes. Adynamia was present in this group of rats 45 minutes after the induction of shock $(n=7)$.

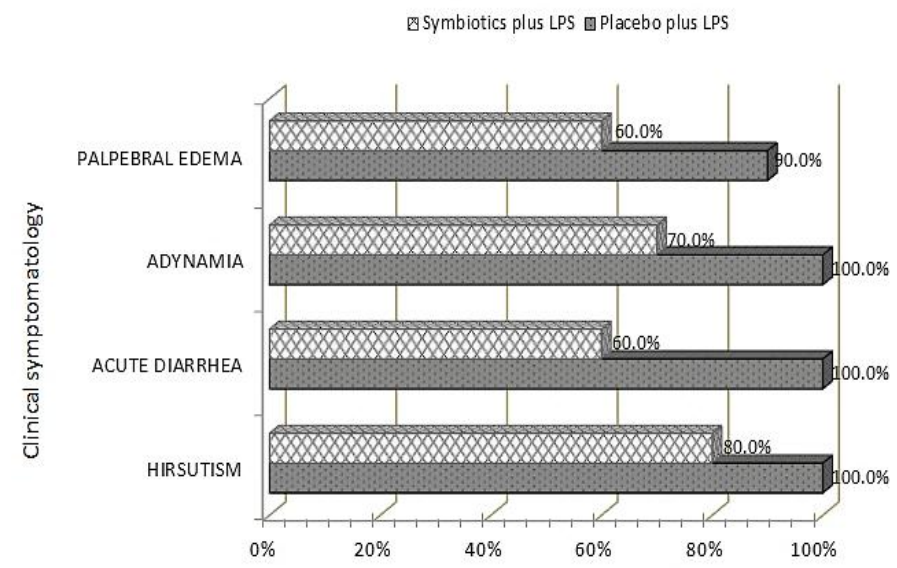

Figure 1: Clinical symptomatology of the rats subjected to the indicated treatments. Clinical symptomatology was registered at 75 minutes after the induction of endotoxic shock for the group of animals under the specified treatments, expressed in percentage ( $n=10$ per group).

\section{Effects of symbiotic supplementation on mortality}

Figure 2 shows mortality among the Placebo plus LPS group and the Symbiotic plus LPS group. In the P + LPS group the first rat death occurred at 9 hours and the last at 20 hours after the induction of endotoxic shock, resulting in $100 \%$ mortality. Death of rats in the S + LPS group was registered at later times with the first death occurring 18 hours after the induction of endotoxic shock, and mortality present in $90 \%$ at 23 hours. However, one animal survived the endotoxic shock beyond the time equivalent to 30 hours (Period of time the trial lasted); therefore, survival rate for this group was 10\% (Figure 2).

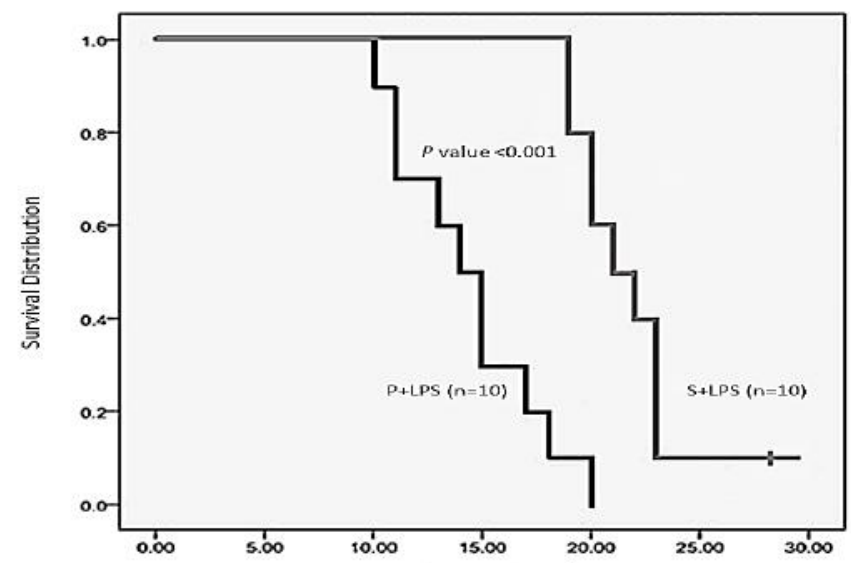

Figure 2: Kaplan-Meier survival plot for male Wistar rats subjected to the indicated treatments. Cumulative survival curves versus time (hours) are shown. This study was conducted over a period of 30 hours.

Evaluating Relative Risk (RR) and statistical analysis for the aforementioned treatments, the P + LPS group had a value of 0.874 . In contrast, RR in the S + LPS group was 0.143 . The difference in these values was statistically significant $(p=0.001)$. This suggests that pre-treatment with symbiotics decreases the risk of mortality, while in the group receiving only placebo the risk of mortality presented in about $87 \%$ of the individuals.

\section{Effects of symbiotic supplementation on quantification of se- rum proinflammatory cytokines}

Figure 3 shows serum levels of proinflammatory cytokines and courses of time between the Placebo and Symbiotics groups in response to a lethal dose of LPS. Baseline levels of proinflammatory cytokines (TNF- $\alpha$ IL-1 $\beta$ and IL-6) were detected in the serum of rats treated with physiologic isotonic saline solution and these levels did not change during the course of experiment. Concentration of TNF- $\alpha$ was significantly increased in the P + LPS group compared to Control group, reaching a peak 4 hours after the treatment $(p<0.001)$ Pre-treatment with symbiotics prevented excessive expression of TNF- $\alpha$ in response to LPS $(\mathrm{p}<0.05)$ (Figure 3A).
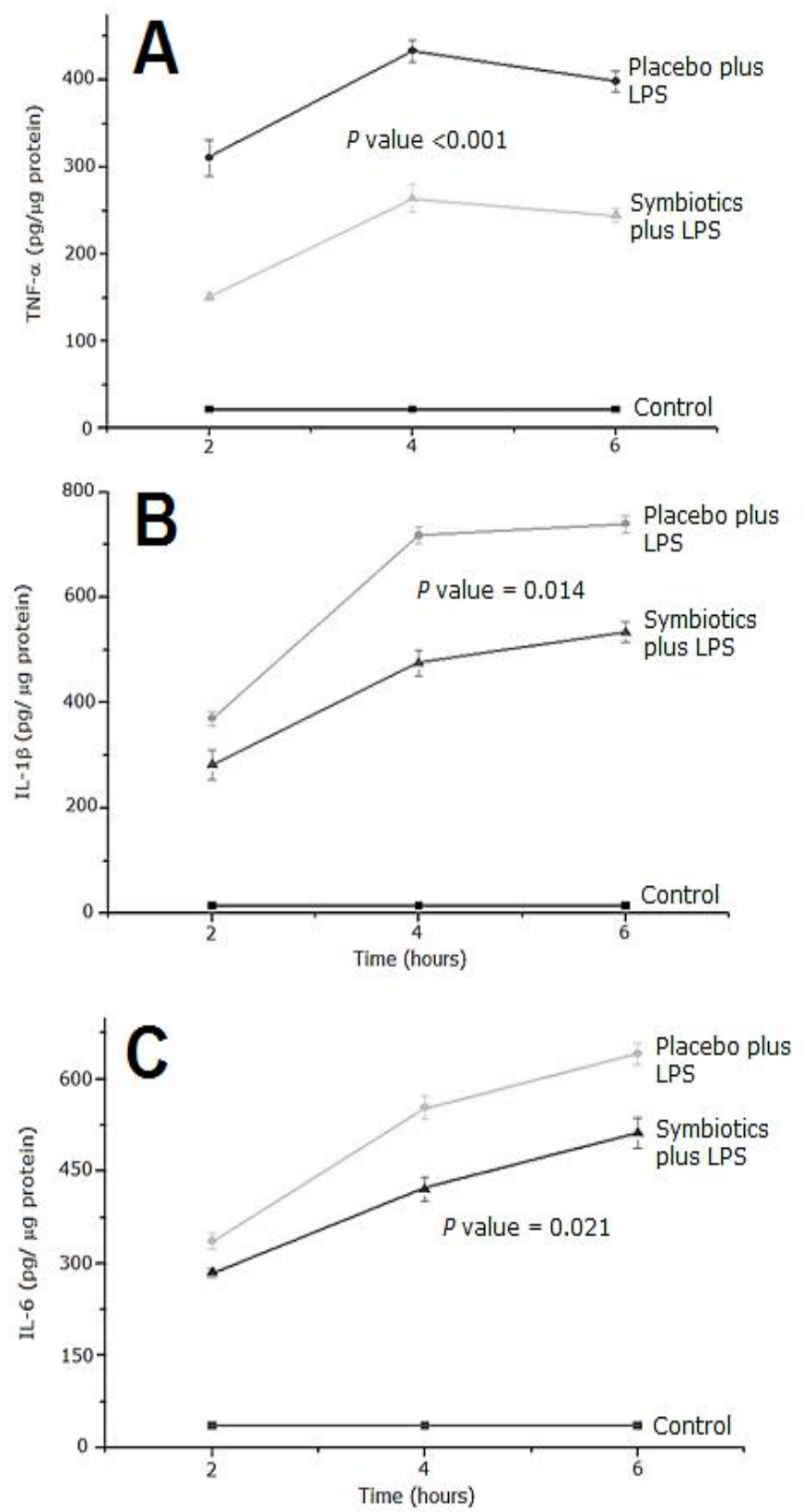

Figure 3: Time course levels of TNF- $\alpha$ (A) IL-1及 (B) and IL-6 (C) in rat serum. Each point represents the Mean \pm SEM. $P$ value $\leq 0.001$ for Placebo plus LPS vs. Control group. 
Placebo plus LPS treatment caused a marked rise in the level of IL-1 $\beta$ compared to Control group (Figure 3B). Concentration of this cytokine for the P + LPS experimental group increased over time and reached a maximum peak at hour 6 , which was significantly different compared to Control group. In contrast, pre-treatment with symbiotics significantly diminished the serum IL-1 $\beta$ values elicited by LPS administration $(p<0.05)$.

As shown in Figure 3C, release of IL-6 increased significantly in response to the LPS challenge reaching a peak at 6 hours. Symbiotics plus LPS treatment elicited a significant decrease in the serum IL-6 concentration ( $p<0.05)$.

\section{Effects of symbiotic supplementation of on membrane flu- idity}

Figure 4A shows that Placebo plus LPS treatment caused a significant decrease of the excimer/monomer ratio of 1,3 dipyrenylpropane (DPyP) in liver samples compared to Control group and the Symbiotic plus LPS group, indicating a decrease of mitochondrial membrane fluidity in the P + LPS group $(\mathrm{p}<0.05)$. In contrast, membrane fluidity values were similar between Symbiotic plus LPS group and the Control group. There were no significant differences in the membrane fluidity of kidney mitochondria between the experimental and control groups (Figure 4B). Mitochondrial membrane fluidity in lungs from the LPS treated groups was lower than Control group ( $\mathrm{p}<$ 0.001) (Figure 4C).
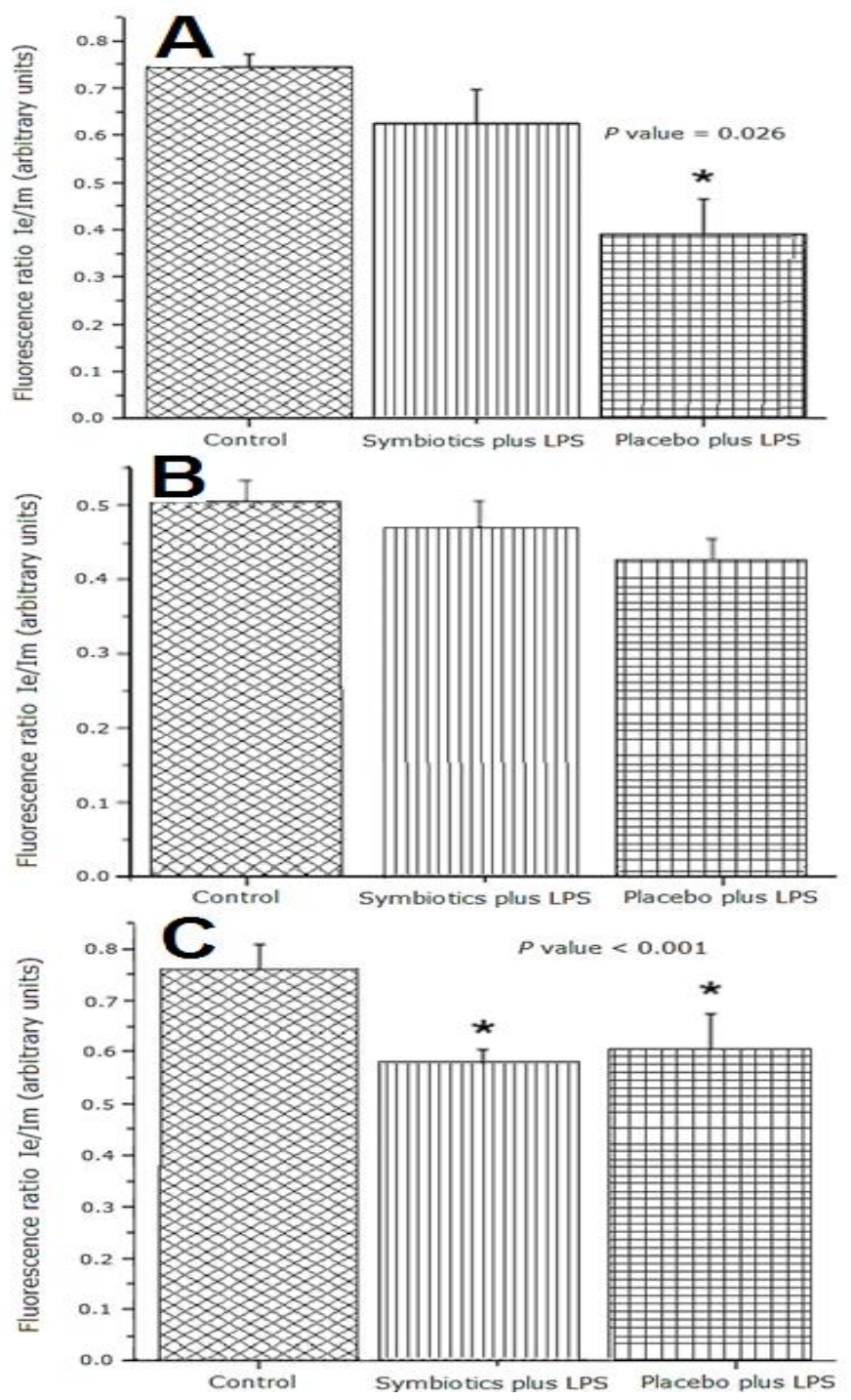

Figure 4: Membrane Fluidity in mitochondria from rat liver (A), kidney (B) and lung (C). Fluorescence ratio of DPyP in mitochondria from rat liver subjected to the indicated treatments. The bar represents the Mean \pm SEM. P value $=0.026$ for Placebo plus LPS vs. Control and Symbiotics plus LPS groups.

\section{Effects of symbiotic supplementation of on enzymatic activi- ty of mitochondrial ATPase}

Figures 5A and 5B show that Placebo plus LPS treatment induced significantly higher mitochondrial ATPase activity in liver and kidney samples compared to Control group $(\mathrm{p}<0.05$ and $p<0.001$, respectively). Symbiotic pre-treatment in rats reduced the ATPase activity to values similar to Control group. No statistical differences were detected in mitochondrial ATPase activity in lung samples in the control and experimental groups treated with LPS (Figure 5C).
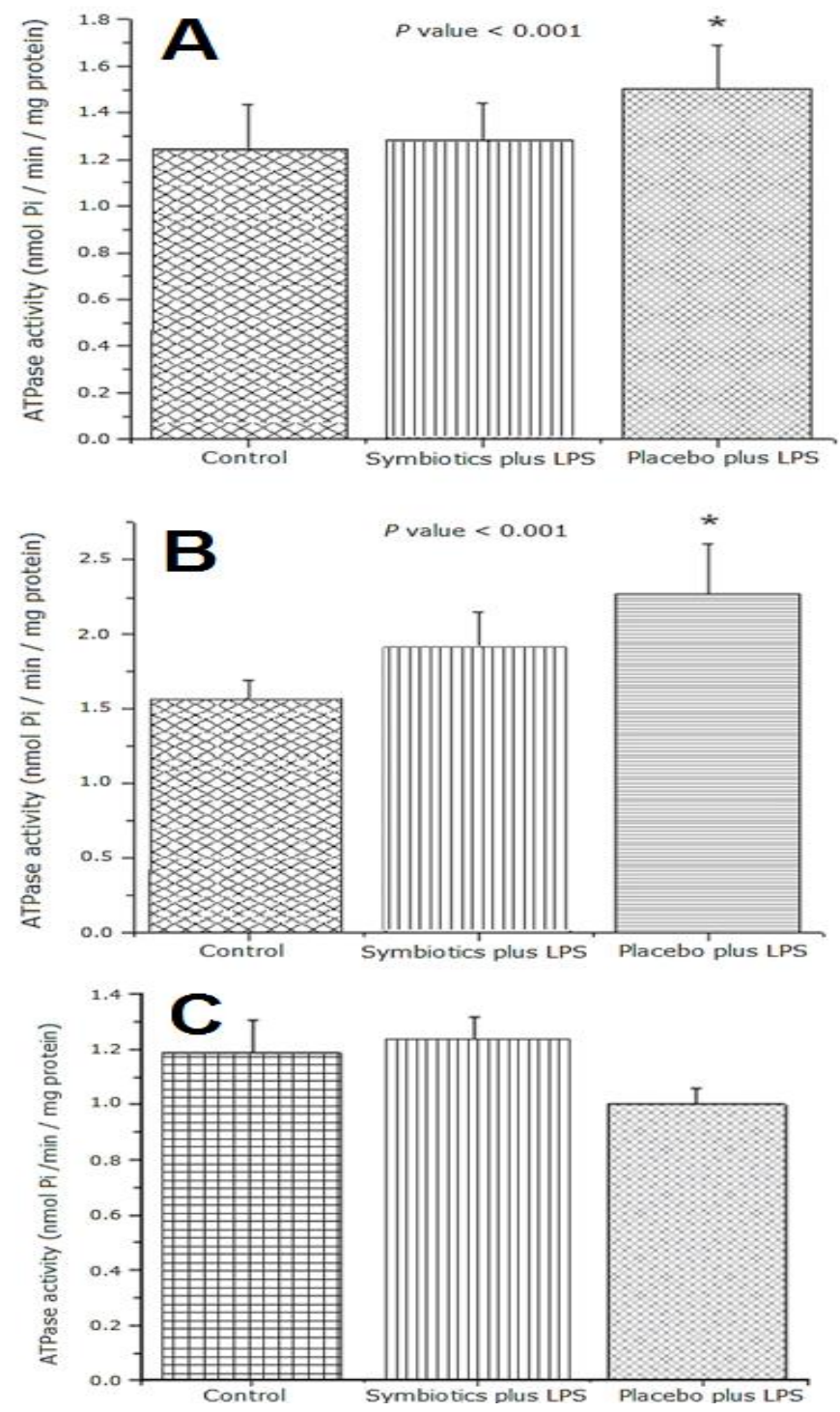

Figure 5: Enzymatic Activity of mitochondrial ATPase from rat liver (A), kidney (B) and lung (C). ATPase activity in mitochondria from rat liver subjected to the indicated treatments. The bar represents the Mean \pm SEM. P value $<0.001$ for Placebo plus LPS vs. Control and Symbiotics plus LPS groups.

\section{Discussion}

Numerous studies have shown that consumption of symbiotics seems like a promising tool for modulating the im- 
mune system ${ }^{[25]}$. Health effects have been documented in epidemiological and interventional studies, especially beneficial effects on the gut microbiota, with important clinical implications in the prevention and/or treatment of infectious and inflammatory diseases ${ }^{[26]}$. Mechanisms include modulation of the functional properties of the microbiota, epithelial, dendritic, and immune cells. But how symbiotics affect the composition of the gut microbiota and its immunological-biochemical relationships, or how they beneficially stimulate other bacteria besides lactic acid, have not yet been studied in depth ${ }^{[27,28]}$, providing opportunity for an important line of research into new strains of pro-biotic and pre-biotic combinations. The beneficial effect of bifidobacteria and lactobacilli is exercised through various mechanisms. These probiotics produce substances that act directly against other harmful bacteria by competing to conquer the intestinal walls to implant there and multiply (Adhesion and colonization $)^{[29]}$. Recent studies suggest that probiotics may inhibit activation of nuclear factor kappa-light-chain-enhancer of activated B cells (NF-kB) in lymphocytes isolated from the lamina propria in samples of intestinal biopsy, and down regulate the secretion of inflammatory cytokines ${ }^{[30-33]}$.

This study shows that intraperitoneal administration of a single lethal dose of LPS from $E$. coli. causes, in the acute phase, the following: hirsutism, acute diarrhea, adynamia, and palpebral edema. It was found that these clinical symptoms were considerably reduced in the Symbiotic plus LPS group; that is, reducing symptomatology up to $32.5 \%$ compared to the Placebo plus LPS group. As well, it improved life condition by diminishing hirsutism in $20 \%$, with this condition presenting at later times; and adynamia and palpebral edema were also reduced in $30 \%$ and $40 \%$, respectively. Comparing these results to a $\mathrm{Ka}-$ plan-Meier survival chart, treatment with symbiotics resulted in longer lifespan, significantly increasing the survival rate up to $30 \%$ at 22 hours after the induction of endotoxic shock, and considering that one animal survived endotoxic shock.

We observed the effect of symbiotics at a systemic and sub-cellular level by measuring proinflammatory cytokine production, mitochondrial ATPase activity, and membrane fluidity. TNF- $\alpha$ levels in the group under the treatment of symbiotics decreased up to $42.2 \%$ compared to the Placebo plus LPS group. IL- $1 \beta$ concentrations were diminished $29.2 \%$ in the group treated with symbiotics versus Placebo plus LPS group. Levels of IL-6 in the group treated with symbiotics were also reduced $20.4 \%$ compared to Placebo plus LPS group. During endotoxic shock oxidative stress generates damage at the cellular level and the activation of macrophages releases proinflammatory cytokines at the systemic and local levels. Production of these cytokines (TNF- $\alpha$, IL-1 $\beta$, IL-6) generates impairment in the epithelial barrier, which can permit bacterial translocation ${ }^{[34]}$; however, it has been described that Lactobacillus rhamnosus exerts a protective effect in the epithelial monolayer, competing for adhesion in the presence of Enterohaemorrhagic E. coli (EHEC). This mechanism of action probably reverses epithelial damage induced by the overproduction of proinflammatory species by inducing tight junction protein redistribution (Claudin-1 and zonula occludens (ZO-1)), preventing damage to the function of the epithelial barrier ${ }^{[35]}$. On the other hand, the decrease in proinflammatory cytokine production can also be attributed to the Lactobacillus inducing the production of IL-12 and IL-10 in macrophages and/ or T-cells, which exerts immunomodulatory properties ${ }^{[36]}$.
The LPS dose used in this experimental model was sufficiently high enough to cause damage at the mitochondrial level. To determine the beneficial effect of symbiotics, analysis of ATPase activity and mitochondrial membrane fluidity were performed. It can be observed that LPS diminished membrane fluidity in the mitochondria of liver, kidney, and lung. Interestingly, pre-treatment with symbiotics restored the membrane fluidity, particularly in liver mitochondria.

Beneficial effects of symbiotics were not observed in lung. It can be hypothesized that the detrimental effect of LPS on lung tissue was not ameliorated by pre-treatment with symbiotics. On the other hand, this study demonstrates that the symbiotic formulation used exerts beneficial effects in the rats subjected to endotoxic shock. These favorable results may be associated to the lower levels of pro-inflammatory cytokines, and restored mitochondrial membrane fluidity and ATPase activity, in the tissues analyzed. It is well known that sepsis causes a disturbed environment, as well as altered microbiota, in the host due to the presence of pathogenic species, and therefore leads to a systemic failure. Inhibition of the pathogens may be due to the mechanism of action of the probiotics, production of antimicrobial agents such as lactic acid and short chain fatty acids such as butyrate, propionate and acetate, and increased competition between pathogens and probiotics for adhesion to epithelial cells, mainly the gut barrier ${ }^{[37,38]}$. It's also well-known that probiotics and prebiotics can prevent apoptosis by enhancing tight junction proteins expression ${ }^{[39]}$; and thus, prevent bacterial translocation, and possibly maintain homeostasis, resulting in protection from damage $^{[10]}$ and potential prevention of the clinical symptomatology, as seen in the results.

Symbiotics have the potential to activate immune cells, such as B-cells and T-cells, releasing them into the bloodstream and modulating the immune system ${ }^{[40]}$.

The challenge for experts working in the medical use of functional foods such as probiotics, prebiotics, symbiotics, and novel foods, consists in the application of new knowledge generated by basic scientists in the field of gut microbiota, and the development of novel therapies using symbiotics to improve treatments of allergy, atopy and inflammatory bowel condition, and possibly prevention in chronic or acute diseases. The probiotic research where gastroenterology, immunology and microbiology intersect is very dynamic in both basic and clinical fields. Pursuit in knowing the complex molecular mechanisms that lead to the efficacy of probiotics could also stimulate the development of greater success in the formation of probiotics ${ }^{[41-43]}$.

\section{Conclusion}

Pre-treatment with the symbiotic formulation improved survival rate, diminished levels of proinflammatory cytokines, and exerted beneficial changes in mitochondrial markers such as membrane fluidity and ATPase activity. Therefore, clinical data showed a beneficial effect from the symbiotic formulation used.

Acknowledgements: The authors gratefully acknowledge the help of Doctor Jose Blanco Fabela (Animal supply, CIBO-IMSS. Guadalajara, Jalisco, México).

Conflict of Interest: No conflict of interest. 


\section{References}

1. Venet, C., Zeni, F., Viallon, A., et al. Endotoxaemia in Patients with Severe Sepsis or Septic Shock. (2000) Intensive Care Med 26(5): 538544.

2. Lee, C.C., Lee, C.H., Chuang, M.C., et al. Impact of inappropriate empirical antibiotic therapy on outcome of bacteremic adults visiting the ED. (2012) Am J Em Med 30(8): 1447-1456.

3. Angus, D.C., Linde-Zwirble, W.T., Lidicker, J., et al. Epidemiology of severe sepsis in the United States: analysis of incidence, outcome, and associated costs of care. (2001) Crit Care Med 29(7): 1303-1310.

4. Park, B.S., Lee, J.O. Recognition of lipopolysaccharide pattern by TLR4 complexes. (2013) Exp Mol Med 45: e66.

5. Toledo-Pereyra, L.H., Suzuki, S. Neutrophils, cytokines and adhesion molecules in hepatic ischemia and reperfusion injury. (1994) J Amer Coll Surg 179(6):758-762.

6. Morgan, E.T. Regulation of cytochromes P450 during inflammation and infection. (1997) Drug Metab Rev 29(4): 1129-1188.

7. Gill, R., Tsung, A., Billiar, T. R. Linking oxidative stress to inflammation: Toll-like receptors. (2010) Free Radic Biol Med 48 (9): 11211132.

8. Liu, D.Q., Gao, Q.Y., Liu, H.B., et al. Probiotics Improve Survival of Septic Rats by Suppressing Conditions Pathogens in Ascites. (2013) World J Gastroenterol 19(25): 4053-4059.

9. Marshall, J.C. Lipopolysaccharide: An Endotoxin or an Exogenous Hormone? (2005) Clin Infect Dis 41(7): 70-80.

10. O'Flaherty, S., Saulnier, D.M., Pot, B., et al. How can probiotics and prebiotics impact mucosal immunity? (2010) Gut Microbes 1(5): 293-300.

11. Khailova, L., Frank, D.N., Dominguez, J.A., et al. Probiotic Administration Reduces Mortality and Improves Intestinal Epithelial Homeostasis in Experimental Sepsis. (2013) Anesthesiology 119(1): 166-177. 12. Braga, M., Ljungqvist, O., Soeters, P., et al. ESPEN Guidelines on Parenteral Nutrition: Surgery. (2009) Clin Nutr 28(4): 378-386.

13. Kaminogawa, S., Nanno, M. Modulation of Immune Functions by Foods. (2004) Evid Based Complement Alternat Med 1(3): 241-250.

14. Pang, G., Xie, J., Chen, Q., et al. How functional foods play critical roles in human health. (2012) Food Sci and Hum Wellness 1(1): 26-60. 15. Perez D. Adición de probióticos y prebióticos a fórmulas infantiles y su efecto sobre la biodisponibilidad mineral. (2003) Fac Veterinaria Murcia pp: 59-94.

16. Capurso, L., Morelli, L. Probiotics, Prebiotics, and New Foods. (2004) J Clin Gastroenterol 42(3): 109-110.

17. Peña, A.S. Intestinal flora, probiotics, prebiotics, synbiotics and novel foods. (2007) Rev Esp Enferm Dig 99(11): 653-658.

18. Bazzocchi, G., Gionchetti, P., Almerigi, P.F., et al. Intestinal microflora and oral bacteriotherapy in irritable bowel syndrome. (2002) Dig Liver Dis 34 (2): S48-S53.

19. Gassull, M.A. Review Article: the Intestinal Lumen as a Therapeutic Target in Inflammatory Bowel Disease. (2006) Aliment Pharmacol Ther 24 (3): 90-95.

20. González-Renovato, E.D., Alatorre-Jiménez, M., Bitzer-Quintero, O.K., et al. Effect of Nutrisim (C) on Endotoxic Shock Induced by Lipopolysaccharide from Escherichia coli: 0111:B4in Rats: Structural Study of Liver, Kidney and Lung. (2013) J Clin Exp Pathol; 4(1): 153.

21. Moreno, R., Torres, M.E. Control of Oxidative Phosphorylation in Mitochondria, Cells and Tissues. (1991) Int J Biochem 23(11): 11631174.

22. Lowry, O.H., Rosebrough, N.J., Farr, A.L., et al. Protein measurement with the folin phenol reagent. (1951) J Biol Chem 193(1): 265275.

23. Ortiz, G.G., Pacheco-Moisés, F.P., Hafidi, M.E., et al. Detection of

Online ISSN: $2377-0619$

Journal Title: International Journal Food and Nutritional Science Journal Short Name: Int J Food Nutr Sci membrane fluidity in submitochondrial particles of platelets and erythrocyte membranes from Mexican patients with Alzheimer disease by intramolecular excimer formation of 1,3 dipyrenylpropane. (2008) Dis. Markers 24(3): 151-156.

24. Sumner, J.B. A method for the colorimetric determination of phosphorus. (1944) Science 100(2601): 413-414.

25. Vieira, A.T., Teixeira, M.M., Martins, F.S. The Role of Probiotics and Prebiotics in Inducing Gut Immunity. (2013) Fron Immunol 4: 445. 26. Romeo, J., Nova, E., Wärnberg, J., et al. Immunomodulatory Effect of Fibres, Probiotics and Synbiotics in Different Life-Stages. (2010) Nutr Hosp 25(3): 341-349.

27. Passeron, T., Lacour, J.P., Fontas, E., et al. Prebiotics and synbiotics: two promising approaches for the treatment of atopic dermatitis in children above 2 years. (2006) Allergy 61(4): 431-437.

28. Bengmark, S. Synbiotics and the mucosal barrier in critically ill patients. (2005) Curr Opin Gastroenterol 21(6): 712-716.

29. Karczewski, J., Troost, F.J., Konings, I., et al. Regulation of human epithelial tight junction proteins by Lactobacillus plantarum in vivo and protective effects on the epithelial barrier. (2010) Am J Physiol Gastrointest Liver Physiol 298(6): G851-G859.

30. Miettinen, M., Lehtonen, A., Julkunen, I., et al. Lactobacilli and Streptococci activate NF-kappa B and STAT signaling pathways in human macrophages. (2000) J Immunol 164(7): 3733-3740.

31. Wehkamp, J., Stange, E.F. A new look at Crohn's disease: breakdown of the mucosal antibacterial defense. (2006) Ann N Y Acad Sci 1072: 321-331.

32. Gronlund, M.M., Arvilommi, H., Kero, P., et al. Importance of intestinal colonisation in the maturation of humoral immunity in early infancy: a prospective follow up study of healthy infants aged 0-6 months. (2000) Arch Dis Child Fetal Neonatal Ed 83(3): 186-192.

33. Gronlund, M.M., Salminen, S., Mykkanen, H., et al. Development of intestinal bacterial enzymes in infants--relationship to mode of delivery and type of feeding. (1999) Apmis 107(7): 655-660.

34. Lui, C., Li, A., Weng, Y.B., et al. Changes in intestinal mucosal immune barrier in rats with endotoxemia. (2009) World J Gastroenterol 15(46): 5843-5850.

35. Quin, H., Zhang, Z., Hang, X., et al. Plantarum prevents enteroinvasive Escherichia coli induced tight junction proteins changes in intestinal epithelial cells. (2009) BMC Microbiol 9: 63.

36. Pathmakanthan, S., Li, C.K., Cowie, J., et al. Lactobacillus plantarum 299: beneficial in vitro immunomodulation in cells extracted from inflamed human colon. (2004) J Gastroenterol Hepatol 19(2): 166-173. 37. Cleveland, J., Montville, T.S., Nes, I.F., et al. Bacteriocins: Safe, natural antimicrobials for food preservation. (2001) Int J Food Microbiol 71(1): 1-20.

38. Lee, Y.K., Lim, C.Y., Teng, W.L., et al. Quantitative approach in the study of adhesion of lactic acid bacteria to intestinal cells and their competition with enterobacteria. (2000) Appl Environ Microbiol 66(9): 692-697.

39. Ewaschuk, J.B., Diaz, H., Meddings, L., et al. Secreted bioactive fac $\neg$ tors from Bifidobacterium infantis enhance epithelial cell barrier function. (2008) Am J Physiol Gastrointest Liver Physiol 295(5): 1025 1034.

40. Guéniche, A., Philippe, D., Bastien, P., et al. Probiotics for photoprotection. (2009) Dermatoendocrinol 1(5): 275-279.

41. Haskey, N., Dahl, W.J. Synbiotic therapy: a promising new adjunctive therapy for ulcerative colitis. (2006) Nutr Rev 64(3): 132-138.

42. Kato, K., Mizuno, S., Umesaki, Y., et al. Randomized placebo-controlled trial assessing the effect of bifidobacteria-fermented milk on active ulcerative colitis. (2004) Aliment Pharmacol Ther 20(10): 11331141.

43. Bohm, S.K., Kruis, W. Probiotics: do they help to control intestinal inflammation? (2006) Ann N Y Acad Sci 1072: 339-350.

Ommega Online Publishers

E-mail: foodscience@ommegaonline.org

Website: www.ommegaonline.org 